\title{
THE PHYSIOLOGICAL BASIS OF RESTITUTION OF LOST PARTS
}

\author{
BY \\ C. M. CHILD \\ Hull Zoölogical Laboratory, University of Chicago, Chicago, Ill. \\ With One Figure
}

In a series of "Studies on Regulation" which have appeared in Roux's Archiv and the Journal of Experimental Zoölogy during the last five years, and in certain other papers (Child 'o6a, 'o6b), I have attempted to point out the essentially functional character of form-regulation and have defined regulation in general as a return or approach to physiological equilibrium after such equilibrium has been disturbed or altered (Child 'o6a). According to this idea form-regulation and functional regulation are both essentially the same thing. It is perhaps unnecessary to state again here what I have repeatedly stated, viz: that the term "functional" is used in this connection in its widest sense as equivalent with "dynamic" or "physiological" and so includes all dynamic factors in organic life. In other words, the problem of form-regulation is a physiological problem and not a problem sui generis as Driesch and various other authors have maintained.

Let us consider for a moment what these assertions imply as regards the factors concerned in the determination of any particular structure. If we assert that a given structure is altered or determined by functional conditions does not this assertion necessarily involve the idea of relation to its environment, intra-organic or extra-organic or both? As a matter of fact the very essence of the term "functional" as employed in these papers is to be found in the interrelation or correlation between the different parts of the organism and between the organism and its extra-organic environment.

The Journat of Experimental Zoölogy, vol. v, No. 4 
That this could fail to be evident to any reader of these papers had not occurred to me until a recent paper by Prof. S. J. Holmes (Holmes '07) came to my notice. This paper is a restatement of the author's symbiotic theory of form-regulation and a reply to certain criticisms of my own (Child 'o6a) of an earlier statement of this theory (Holmes ' ${ }_{4}$ ). Holmes maintains that my suggestions concerning the nature of form-regulation do "not contain any general principle of explanation for that functional substitution and equilibration upon which it is assumed that form-regulation depends. But I suspect that when his theory comes to be developed so as to supply this missing element it will involve the assumption of some such symbiotic relation between the parts of the organism as I have assumed " (p. 424). If I understand this assertion, it involves a serious misapprehension of my position. I have insisted again and again in my work on form-regulation in the interrelations or correlations between parts--in fact, certain of my papers have been concerned chiefly with showing that such relations existed. Moreover, it is in consequence of the existence of such relations that I regard form-regulation as essentially a functional process. Even in my earliest papers positive statements on this point were made. Thus, for example, on p. 219 of No I of my Studies on Regulation (Child 'O2) in a consideration of the general body-form of Stenostoma I wrote: "Every organism is what it is because of the relation of all its parts to each other and to the rest of the world. If any of these relations are changed the organism is changed." And again in No. IV of the Studies (Child 'O4a) in a discussion of "formative factors:" "All the complex activities of which organisms are capable are "formative factors:' when we can view all of these in their complex interrelations, then and then only shall we "understand' organic form." Also in No. V (Child 'O4b): "The factors of organic form include all the activities of organic substance as well as the environmental factors in varying degree. Indeed, in most cases, if not in all, we may regard organic form as the visible effect upon the protoplasm of functional factors in the widest sense" (pp. 468-469). In the later papers these interrelations are still more strongly emphasized. I have preferred not to designate them as symbiotic relations since 
I cannot see that anything is gained by the use of this term. Moreover, it seems to me that many of the correlations are not really symbiotic at all, except in so far as they may be mutual with respect to complex parts of the organism. For example, the mere mechanical union with other parts is undoubtedly in many cases one factor in preventing parts of the organism from undergoing regulation into wholes. But $I$ cannot see that it serves any useful purpose to call such factors symbiotic relations. It seems preferable therefore to maintain that these relations, or as I believe we may more properly call them, correlations, are physically and chemically of all sorts possible in the material and environment in which they exist. Moreover, while many of them are undoubtedly mutual,i. e., reciprocal, at least as regards complex parts, others are just as certainly largely or wholly one-sided so far as form is concerned. It seems scarcely necessary to enlarge further upon this point. As regards the existence of relations between parts as an essential feature of regulation Holmes and I agree perfectly. As regards form-regulation we differ, in that it seems to me difficult or impossible to account for the facts on the basis of symbiotic relations, even in the widest sense.

Holmes' illustration of the process of regeneration is as follows: "Let us imagine an organism made up of a number of differentiated cells, each of which derives some advantage from some substances produced by the contiguous cells, and giving out some substance upon which the contiguous cells are more or less dependent. We will suppose that in addition to these differentiated cells, there are scattered through the body numerous indifferent or embryonic cells whose multiplication is held in check by the others, but which upon the removal of any part respond to the functional disturbance by growth and multiplication near the place of mutilation. We may represent our organism by the following diagram in which the differentiated cells are represented by the larger circles $A, B, C$, etc., and the indifferent cells by the smaller circles between them. Each cell such as $A$ contributes something utilized by $B, G$, and $F$, and derives something in return from each of these sources. Now suppose $A$ is removed: the indifferent cell lying nearby, no longer held in check by the same stimuli, 
begins to grow and develop. What line of differentiation will it most naturally take? Owing to the symbiotic relation existing between the cells differentiation in the direction of $A$ will be most favored as this secures it the advantages which $A$ received. In other words, this will be the direction of development along which social pressure will tend to guide it. And the result will be a regeneration of the missing part" (Holmes '04, p. 282; '07, pp. 420, 4 II).

In 1906 (Child '06a) I criticised this illustration on the ground that if the cells or parts were mutually dependent, i. e., if symbiotic relations existed between them, removal of any one of them, e. g., $A$, would bring about changes in the others in consequence of which their influence upon the undifferentiated cell substi-

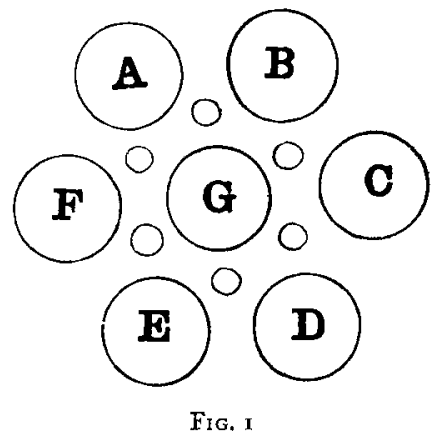

tuted for $A$ would be different from what it was originally, and hence the undifferentiated cell or part would develop-not into another $A$ but into something else. There is logically no escape from this conclusion. The removal of $A$ results in the formation of a new system different from the original and must necessarily do so, except under certain limiting conditions to be discussed below.

Holmes' reply to my criticism is as follows: "According to Child, since the removal of $A$ would alter $B, G, F$, etc., not only something different would be developed in place of $A$, but the whole complex, according to my theory, would be profoundly altered. How far this tendency will result in a modification of these cells depends on the plasticity of the organism and the degree 
of mutual dependence of the parts-factors of course which vary in different organisms. But Child overlooks the fact that according to the symbiotic relation assumed, the other cells $C, D, E$, etc., tend to keep $B, F, G$ in their original condition. In so far as these remain in their original state, their influence on the indifferent tissue in the region of $A$ will tend to mold it in the direction of the missing parts. In so far as $B, G$ and $F$ are modified through the loss of the missing part, their influence on the tissue in the region of $A$ will come to be modified, and they will in turn modify the cells lying next to them. But, as there is a tendency for the modifications produced by the loss of $A$, to spread successively to other parts, there is also a tendency, according to my theory, toward the checking and reversal of this process. If the loss of $A$ tends to modify $B, F$ and $G$, the presence of $E, C$ and $D$ tends to hold them in place, and in so far as these are maintained through this influence they tend to mold the tissue in the position of $A$ into the form of the missing part; and in so far as this is so molded, its modifying influence on $B, F$ and $G$ is diminished" (Holmes 'o7, pp. 425, 426).

I am unable to see that this argument shows that something like $A$ may be generally replaced. Undoubtedly the modifying influence of $A$ upon the contiguous cells or parts $B, F, G$, is lessened by the presence of other cells or parts, $E, C, D$, but it is not reduced to zero in any case where the relations between parts are mutual. The balance between the "tendency for the modification produced by the loss of $A$ to spread" and the opposite tendency simply determines how great or how small the modification shall be. Something more or less like $A$ may undoubtedly be produced in many cases, but according to this hypothesis we should expect that the regenerated part would differ more or less widely from the original part in most cases.

In fact, if the restoration of a part removed is purely a matter of interrelation between the various parts of the system, we must modify this hy pothesis in either one of two ways to account for it. First: we may assume that the removal of the part, $A$ in Holmes' diagram, does not alter the other parts, $B, G, F$, etc., in any way which affects essentially their interrelations with the parts of the system. 
In this case the undifferentiated material which in the absence of $A$ is stimulated to develop, will develop into another $A$. But in this case the relation between the original $A$ and the other parts of the system is essentially one-sided and not mutual or symbiotic. Or as a second possibility, we may assume that the relation between $A$ and the other parts of the system is such that removal of $A$ produces modifications in the other parts only very slowly, while in the absence of $A$ these other parts affect the undifferentiated cells in such manner as to bring about rapid development so that restoration is complete before the parts $B, G, F$, etc., have been appreciably altered by the absence of $A$. Here the relations, though in the final analysis mutual, are so far as $A$ and its restoration are concerned, one-sided. In short, if we accept the symbiotic theory as a basis, we can account for the restoration of a part like that removed only by additional assumptions, according to which the relations involved in the restoration become practically one-sided rather than mutual.

In my earlier criticism of Holmes' theory (Child 'o6a, pp. '420, $42 \mathrm{I})$ the following statement appears: "To return to Holmes' diagram, replacement of $A$ can occur only when the relation is largely one-sided, i. e., when $A$ is dependent on $B-F$, but these latter are not to any marked degree dependent on $A$. In this case, and in this case only, will the "social pressure" force the undifferentiated cell to differentiate into something like $A$."

Holmes replies to this: "Where redifferentiation from new tissue is concerned, as in the present case, it is not the relation of $A$ to $B-F$, that should be more or less one-sided, but the relation of the tissue in place of $A$ to this complex. This is an important distinction which Child does not seem to have considered. $B-F$ are relatively fixed, the tissue in place of $A$ is young and plastic, and more dependent so far as the direction of its differentiation is concerned, upon $B-F$, than these are upon it. We may grant that when regeneration occurs, the relation of dependence between the old parts and the new tissue is more or less one-sided, although the relations of the part removed may not have been. This would naturally result if the parts were relatively stable. They may be in a symbiotic relation, nevertheless, each part contributing in 
some way to the normal functioning of the others, and dependent to the extent that the removal of one part may alter only to a certain degree the quality and quantity of the activity of the surrounding parts, without producing extensive modification of structure or function" (Holmes '07, pp. 426, 427).

The first part of this argument seems to me to obscure the real point at issue. If the relation between $A$ on the one hand and $B-F$ on the other is not at least largely one-sided, removal of $A$ must alter $B-F$, and if, as Holmes assumes, the new tissue which replaces $A$ is more dependent on $B-F$ than they on it, it becomes still more difficult to understand how the new tissue can replace $A$, for, so far as $B-F$ are concerned, it does not at first take the place of $A$ functionally. In the last sentence quoted, Holmes attempts to save his symbiotic theory after admitting that in regeneration the relation may be more or less one-sided, by suggesting the existence of symbiotic relations which do not produce "extensive modifications of structure or function" when one part is removed. It seems to me that such relations are negligible quantities so far as form-regulation is concerned, for if removal of a part of the complex does not produce extensive modifications of structure or function in the parts remaining, we must certainly conclude that the presence of this part is not essential for the maintenance of the characteristic structure and function in the other parts. Evidently then this assumption does not relieve us from the necessity of assuming that the remaining parts are, so far as form and structure are concerned, practically independent of the part removed, i. e., that the relations involved in form-regulation are largely one-sided in cases where restoration of the missing part occurs. It makes no difference whether we regard the persistence of $B-F$ in essentially unchanged condition after the removal of $A$ as due to "relative stability" or to real independence of $A$. The fact remains that $A$ can be restored only in case the other parts do persist essentially unchanged during the period between its removal and its restoration to a certain stage of development. And it is just as certain that Holmes' symbiotic hypothesis cannot account for such persistence except by assuming the existence of special conditions which modify the relations between parts so 
that they become essentially one-sided rather than mutual. If the restoration of a part like that removed were the exception rather than the rule, or even if it were less frequent, we might still accept the hypothesis. But a hypothesis which can account for the typical phenomena within its field, only with the aid of additional special assumptions, which in this case amount practically to throwing over the hypothesis, can scarcely be regarded as satisfactorv.

The numerous cases already known where an animal is capable of replacing a part repeatedly after successive removals seem to me to furnish additional evidence against Holmes' theory. Even so important a part as the head may be replaced repeatedly in many forms without appreciable change in character. It is scarcely probable, to say the least, that the relations between the head-region and other parts are one-sided in the sense that it is dependent on them, while they are independent of it. And if the relation is not one-sided in this sense, we should expect that repeated removals of the head would bring about essential changes in the other parts, even if the first removal did not. If such changes in the other parts do occur to any marked extent, it is difficult to understand how a new head like the old can be replaced time after time as the result of "social pressure," for such changes in the old parts must alter the character of the "social pressure." Here then Holmes' theory leads us into something closely approaching a dilemma.

Holmes continues: "If the parts $B-F$ were more plastic, absence of $A$ would naturally tend to cause greater changes in them, especially if new tissues were not produced in place of $A$, which would come to assume some of the missing functions before the modification extended very far. There would then be a progressive modification extending from the region of $A$, which would tend to become less the farther it extended, but eventually perhaps affecting more or less the entire organism. Functional equilibrium would then be maintained by working over the organism so that all the parts were adjusted to functioning on a smaller scale. The different methods of regulation, through morphallaxis, regeneration and the various combinations of these proc- 
esses are, I believe, interpretable according to the symbiotic theory, and the relations of regeneration and morphallaxis to the degree of specialization of the parts which Child has elaborated, are, in fact, exactly what the theory would lead us to expect" (Holmes 'o7, p. 427).

Here Holmes fails absolutely, so far as I can see, to explain how and why equilibrium will be maintained. Certainly the "progressive modifications" resulting from the removal of $A$ cannot bring the system back to its original condition: they must lead either to destruction of the system, or rather of the parts of it which remain, or else to a new condition of equilibrium different from the old. How does Holmes know that "functional equilibrium would then be maintained by working over the organism so that all the parts were adjusted to functioning on a smaller scale?" What factor in the parts remaining compensates for the "progressive modifications" resulting from the loss of $A$ ? Why should there be any compensation? To none of these questions does Holmes' hypothesis give any answer.

According to the symbiotic theory as Holmes has presented it, the removal of a part is, at least in many cases, analogous to removal of a quantity from one side of an equation without change in the other. It is obvious that such procedure alters the value of one side of the equation in all cases except where the quantity removed is equal to zero.

In short I believe that Holmes' theory of regulation overlooks the most essential feature in the process of replacement of a part removed. This feature is the qualitative functional totipotence of the remaining parts after removal of the part in question. In other words, a part which has been removed cannot be replaced unless something remains after its removal which plays its part functionally in some degree. According to Holmes' theory its place is taken by undifferentiated tissue, which is forced to develop into something like the part removed by the influence exerted upon it by other parts. But this undifferentiated tissue cannot exert the same influence on other parts as was exerted by the part removed. Moreover it is difficult to understand how undifferentiated tissue whose differentiation is held in check by the other 
differentiated parts, could persist in a system such as Holmes postulated. If it has no function in the system and is not in symbiotic relation with other parts, why should it not disappear? If, on the other hand, symbiotic relations between it and other parts exist, it should, according to Holmes, differentiate into something. It is evident therefore that something besides undifferentiated tissue must take the place functionally of the part removed if replacement is to occur.

We can most readily gain an idea of what this something is by means of a concrete example. In Planaria and various other triclads, where even small pieces are capable of replacing all parts, we find that the reactions of such pieces, while differing in degree from those of the original animal, do not differ essentially in kind After removal of the head, for example, the piece reacts in much. the same manner as when the head was present, though more slowly and with less energy. In Leptoplana, on the other hand, where regeneration of a head does not occur after removal of the ganglia, the piece without ganglia is at once and clearly distinguishable from the animal with ganglia by the character of its reactions.

In Planaria then, and in the orher forms where replacement of the head and ganglia are possible, the piece still retains in some degree the functional characteristics of a head-region. In removing the head we have not removed the only region possessing such characteristics, but only the region which possesses them in the highest degree of any part of the animal. In Leptoplana removal of the head and ganglia leaves no part which can supply functionally, even in slight degree, their place, and formation of a new head is impossible.

We must conclude that the localization of visible structural differentiation in an organism is not necessarily coextensive with the localization of functional processes or conditions characteristic of this region, but may be limited to the region of greatest energy of these processes or conditions. It is a well-recognized fact that the so-called functional structure of bone, tendon, etc., represents only the most frequent or most energetic functional conditions, and there is every reason to believe that similar relations ex st between structure and function in many other cases. The case of Planaria 
cited above is in fact a demonstration that functional processes may be less sharply localized than the structures which represent them. The anatomical structure known as the head in Planaria is not the only region where "head-reactions" are possible, but it does represent the region where they occur with greatest energy and frequency in the normal animal. Admitting this, the question arises as to why heads do not form all along the body in Planaria, i. e., as to why structure should be thus more narrowly localized than function. The answer is not far to seek. If two parts, one of which is capable of reacting in a certain manner more rapidly and with greater energy than the other are correlated, the reaction to a given stimulus will occur in the first part earlier and with greater energy than in the second. The fact that a reaction has occurred in the first part must bring about changes in the system in consequence of which the character of reaction in the second part is altered. If structure is, as I believe, the visible expression of functional or dynamic conditions, we cannot expect that the second part, even though it possesses in some degree the same functional capacities as the first should exhibit the same structure, for the very fact of its correlation with the first part which possesses these capacities in greater degree determines that the functional conditions in it shall be different from those in the first part. In general terms we may say that the region where a particular functional complex occurs with greatest energy, frequency or rapidity dominates so far as this particular complex is concerned all other parts of the organism which possess the same capacity in less degree, and modifies their activities to a greater or less extent. Consequently the structure with which a particular functional complex is associated in the normal animal may be much more narrowly localized than the functional complex. In Planaria, for example, the head-structure is limited to the anterior end of the animal, while the functional capacities commonly regarded as characteristic of the head exist at all levels of the body. These other regions are capable of producing a head-structure, but only when isolated from the original head.

It is evident then from this consideration that localization of visible structure is not necessarily an exact criterion of localization of 
functional capacity. In all cases where a difference in localization exists, structure is more narrowly localized than functional capacity. On this fact, which I believe to be of fundamental importance for the problem of form, depends the ability of a part to become a whole when isolated.

In order to bring out clearly the difference between Holmes' hypothesis and my own, we may make use of Holmes' diagram (Fig. I). According to my hypothesis, the various parts, $A, B$, $C, D$, etc., though perhaps visibly different as regards structure, each possess the physiological properties of the others or of some of the others in some degree. $B, G$ and $F$, for example, the parts contiguous to $A$, are capable in some degree of activities similar to those characteristic of $A$, but as long as $A$, a region of greater energy or frequency or rapidity as regards these particular activities is present the correlations arising from it obscure, inhibit or modify the activities of $B, G, F$, so that they appear structurally and functionally to be different from $A$. But when $A$ is removed, the parts $B, G, F$ become at once the dominating parts as regards the $A$-activities and the correlations between them and other parts become similar in kind, to those which previously existed between $A$ and the other parts, though probably different in degree. In short $B, G, F$, or certain portions of them are substituted functionally for $A$ simply because in the absence of $A$ their activities must, by virtue of their constitution, be somewhat similar to those of $A$. No entelechy or other peculiar principle is needed to guide or determine this substitution. It occurs with the same certainty as any other physical phenomenon in all cases where these parts possess the functional capacities to which attention has been called above. According to this hypothesis, the undifferentiated cells postulated by Holmes are not only unnecessary, but could not substitute for $A$ if present, because the parts $B, G, F$ are more like $A$ than are the undifferentiated cells and would therefore dominate in the process of substitution.

The $A$-processes are undoubtedly in most cases, if not in all, at first less energetic or less rapid or both, than they originally were in $A$, and in consequence of this difference the system may regain its original condition of equilibrium in either one of two ways. If 
the other parts $C, D, E$ are plastic, i. e., if their activities are readily and rapidly altered by altered conditions, they will be affected by the decrease in $A$-correlations following the removal of $A$ and will undergo more or less change in response to the changed correlations, i. e., regulation by what we ordinarily call redifferentiation will occur. If, on the other hand the parts $C$, $D, E$ are relatively stable, i. e., not rapidly changed by altered conditions, they and the correlations arising from them will remain much the same as before the removal of $A$. But the $A$-processes in $B, G, F$ are out of proportion to these correlations and must be quantitatively increased by them. In this case then equilibrium is regained by functional hypertrophy of the portions of $B, G, F$, which are the functional substitutes for $A$. This is what we know as regeneration in the stricter sense, i. e., formation of new tissue from the regions adjoining the cut surface and its visible differentiation with increase in size into a part like that removed. In most plants and in some animals regeneration occurs from regions more or less distant from the cut surface, simply because these regions are physiologically more like the missing part than is the region at the cut surface.

As a matter of fact, since correlations in the system are at least in large measure mutual, most if not all cases of restitution are mixtures of redifferentiation and regeneration. Some change, i. e., some redifferentiation occurs in some or in all parts of the system and some regeneration, i. e., functional hypertrophy of the part which forms the physiological substitute for the part removed takes place.

Holmes' hypothesis fails to recognize the fundamental fact, viz: that something must remain after the removal of a part, $A$, which can take its place functionally in the system in some degree. Without this the only factors which can prevent progressive departure from the original condition are lack of plasticity in the parts remaining or one-sided relations between parts. As a matter of fact however plasticity is a conspicuous feature in manv forms in which the regulation of parts into wholes occurs most readily, and on the other hand all the evidence indicates that correlations are in large measure mutual rather than one-sided. In those cases. 
where a part after isolation is incapable of becoming a whole, while the remaining parts are capable of replacing it, there is reason for believing that the correlations are more or less onesided, i. e., the part in question has been so greatly modified by the past or present correlations arising from other parts that it has lost its totipotence and can never become a whole, but the correlations arising from this part have not been sufficient to modify the other parts of the system to an equal extent. Examples under this head are the appendages of arthropods, amphibia, etc.

One other point discussed by Holmes requires brief consideration: in his first paper he selected the regulatory development of a head in Planaria as an illustration of the working of social pressure. In his discussion of this case differentiation is regarded as proceeding from the cut surface distally, in consequence of the social pressure exerted on the new parts by the old (Holmes ' 04 , pp. 282, et seq.). In my criticism of this point (Child 'o6a, p. $42 \mathrm{I}$, et seq.), I called attention to the fact that in Planaria, and in other forms as well, differentiation of the regenerating tissue actually proceeds in the opposite direction, i. e., from the tip toward the base. In reply to my criticism Holmes ('o7, pp. 427, 428) points out that the first visible differentiation is not necessarily the first actual differentiation, that "before any external features are produced in the development of a limb the main outlines of its differentiation may have been established through influence proceeding from its basal part, after which the tip might differentiate more rapidly than the intervening portion and the other visible features of structure appear successively toward the base." He also points out that in many cases the visible differentiation is centrifugal rather than centripetal and cites the case recently described by Zeleny ('07) of the antennule of Mancasellus, in which visible differentiation at first proceeds from the base toward the tip, but later in the opposite direction. He continues: "But granting that, in many cases, differentiation actually begins at the extremity and works toward the base of the regenerating organ, the process is not inconsistent with the point of view here set forth. We may suppose that the influence of the environment causes the extremity of an organ to begin to differentiate 
like that of the missing part. That is only one step. We have then to account for the numerous coördinated differentiations that take place as the part develops toward the base. $* * *$ The fact that, with few exceptions, such as the failure to regenerate the intermediate segments of the appendages, etc., the whole organ, nothing more nor less, is regenerated, and forms a congruent union with the basal part, is indicative of close interaction of the various parts of developing organs with the body of the organism at all stages of the process.

"I am inclined to think that neither centrifugal nor centripetal differentiation, expresses the entire truth of the matter, but that the new part differentiates as a whole, much as organs do in embryonic development, and at all times in intimate functional relations with the old part, differentiation becoming accelerated in one part or another, according to special conditions" (Holmes '07, pp. 428, 429).

As regards most of these points my position does not differ very widely from that of Holmes. My criticism of his analysis of the case of Planaria was directed primarily, not at his hypothesis in general but merely at his failure to consider the actual facts in that case. I see no reason why the occurrence of differentiation in either direction or in both should constitute a fatal objection to his hypothesis or to my own, for such differences are merely incidental and depend on the conditions in individual cases. When my criticism was written the experimental data seemed to indicate that visible differentiation in the centripetal direction was the general rule, though by no means without exceptions, and since Holmes did not in his first paper attempt to account for this fact in any way, his hypothesis was open to criticism. I certainly had no intention of maintaining that differentiation must in all cases proceed centripetally, since at that time various cases were known to me in which visible differentiation proceeded centrifugally. ${ }^{1}$ I do not believe however that Holmes' suggestion that

\footnotetext{
${ }^{1}$ In his discussion of the direction of differentiation in the antennule of Mancasellus, Zeleny ('o7, p. 335) says: "Child has recently expressed the opinion that differentiation must in every case proceed from the tip toward the base and in no other way." My actual statement was that differentiation from the tip toward the base is "a general ruel in cases of regeneration." This statement as it stands is
} 
the new part differentiates as a whole, much as organs do in embryonic development is universally applicable. There are certainly many cases in which the terminal portions attain or approach their final condition of functional activity before the basal parts are formed, and in a considerable number of cases also the basal parts are replaced incompletely or not at all. In fact it seems to me that such cases might be expected to occur, for in a correlated system the conditions for the regulatory formation of non-terminal regions must, at least sometimes, be largely dependent on the existence of typical functional conditions in terminal parts. If conditions in the terminal parts are more important than those in the old parts as determining factors in the differentiation of intermediate parts we should expect to find the intermediate parts differentiating later than the terminal parts, but if, on the other hand, conditions in the old parts are the chief determining factors, differentiation might occur wholly in the centrifugal direction.

Moreover, although I agree with Holmes that the absence of visible differentiation does not necessarily imply absence of physiological differentiation; I am incined to believe that the direction of progression of visible differentiation is not without significance as an indication of the direction of progression of physiological differentiation. In other words, while the absence of visible differentiation proves little or nothing with regard to physiological differentiation, its presence may prove something. I think it probable therefore that in some cases the regenerating part is not differentiated as a whole, but that its various regions are determined successively in one direction or the other: in other cases it may perhaps be differentiated as a whole. It would appear that none of these possibilities conflict with either Holmes' hypothesis or my own.

\footnotetext{
undoubtedly open to misinterpretation and should have been qualified, for I was well aware at the time it was made that centrifugal differentiation occurred in various cascs. In fact, I bad shown in earlier papers (e. g., Child, 'O4b) that the differentiation of the intestine in regenerating parts of Leptoplana is apparently centrifugal. However I take the present opportunity to make acknowledgments to Holmes and Zeleny for calling my attention to this misleading statement, and also to make clear my real position in the matter, which is that differentiation may occur in either direction or in both according to conditions in the particular case.
} 
To sum up: Holmes and I agree in that we both postulate a condition of physiological equilibrium, or rather, as I should put it a condition of oscillation or cyclical change about equilibrium, as the basis of our hypotheses. The chief point of difference between us is that Holmes' hypothesis does not, as I understand it, provide for the maintenance of or return to the typical condition, except by the assumption of relations largely one-sided, or that of lack of plasticity. While these assumptions may serve for certain individual cases, they seem to me to be totally inadequate for the analysis of form-regulation in general. According to my own hypothesis a part can be replaced only when some other part is physiologically sufficiently similar to it to perform its functions at least qualitatively, if not quantitatively, after its removal.

The independent formulation of two hypotheses of form-regulation so similar in general point of view as are Holmes' and my own, is I believe not without significance, since agreement between different observers as regards the general nature of problems may be an indication that real progress in the analysis of data is being made. It is desirable in such cases, and particularly in fields where the data are so varied and complex, that differences of opinion should be fully and critically discussed. For this reason I have ventured to consider at some length in the present paper the points which seem to me debatable, and to state my own position in a manner which I hope will lessen the chances of future misunderstanding.

Hull Zoölogical Laboratory

University of Chicago

February, 1908 


\section{BIBLIOGRAPHY}

Child, C. M. '02-Studies on Regulation. I. Fission and Regulation in Stenostoma. Arch. f. Entwickelungsmech., Bd. xv, H. 2 and 3, I902.

'o4a-Studies on Regulation. IV. Some Experimental Modifications of Form-Regulation in Leptoplana. Journ. Exp. Zoöl., vol. I, no. I, I 904 .

'O4b-Studies on Regulation. V. The Relation between the Central Nervous System and Regeneration in Leptoplana: Posterior Regeneration. Journ. Exp. Zoöl., vol. I, no. 3, 1904.

'o6a-Contributions toward a Theory of Regulation. I. The Significance of the Different Methods of Regulation in Turbellaria. Arch. f. Entwickelungsmech, Bd. xx, H. 3, 1906.

'o6b-The Relation between Functional Regulation and Form-Regulation. Journ. Exp. Zoöl., vol. iii, no. 4, 1906.

Holmes, S. J. 'O4-The Problem of Form-Regulation. Arch. f. Entwickelungsmech. Bd. xvii, H. 2 and 3 , I904.

'o7-Regeneration as Functional Adjustment. Journ. Exp. Zoöl., vol. iv, no. 3 , I907.

Zeleny, C. '07-The Direction of Differentiation in Development. I. The Antennule of Mancasellus macrourus. Arch. f. Entwickelungsmech, Bd. xxiii, H. 2, I907. 